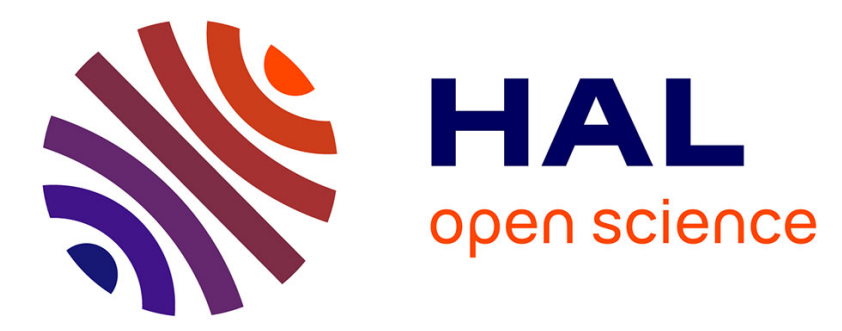

\title{
Étude en microscopie à balayage des dégâts d'irradiation causés dans LiF par bombardement ionique
}

\author{
A. Perez, J. Davenas, P. Thevenard, C.H.S. Dupuy, P Morin, E. Vicario
}

\section{To cite this version:}

A. Perez, J. Davenas, P. Thevenard, C.H.S. Dupuy, P Morin, et al.. Étude en microscopie à balayage des dégâts d'irradiation causés dans LiF par bombardement ionique. Revue de Physique Appliquée, 1976, 11 (1), pp.127-130. 10.1051/rphysap:01976001101012700 . jpa-00243953

\section{HAL Id: jpa-00243953 https://hal.science/jpa-00243953}

Submitted on 1 Jan 1976

HAL is a multi-disciplinary open access archive for the deposit and dissemination of scientific research documents, whether they are published or not. The documents may come from teaching and research institutions in France or abroad, or from public or private research centers.
L'archive ouverte pluridisciplinaire HAL, est destinée au dépôt et à la diffusion de documents scientifiques de niveau recherche, publiés ou non, émanant des établissements d'enseignement et de recherche français ou étrangers, des laboratoires publics ou privés. 


\title{
ÉTUDE EN MICROSCOPIE A BALAYAGE DES DÉGÂTS D'IRRADIATION CAUSÉS DANS LiF PAR BOMBARDEMENT IONIQUE
}

\author{
A. PEREZ, J. DAVENAS, P. THEVENARD, C. H. S. DUPUY
}

(Groupe de Radiolyse et Radiométrie des Matériaux)

et

\section{P. MORIN, E. VICARIO}

(Groupe de Microscopie Electronique quantitative)

Département de Physique des Matériaux associé au C. N. R. S. Université Claude-Bernard Lyon I, 69621 Villeurbanne, France

\begin{abstract}
Résumé. - Un microscope électronique à balayage équipé d'un système de décharge pour l'observation des échantillons isolants nous a permis d'étudier des cristaux de $\mathrm{LiF}$ bombardés avec des protons de $2 \mathrm{MeV}$ et des ions chlore de $56 \mathrm{MeV}$. La zone irradiée présente un contraste clair qui permet une mesure directe des profondeurs de pénétration des ions. Le contraste peut être attribué à la présence des Centres colorés du type $\mathrm{F}$ et agglomérats de $\mathrm{F}$ qui sont également révélés par l'étude des spectres d'absorption optique. Les ions implantés peuvent être également révélés en fin de parcours par l'intermédiaire du contraste chimique dû à la variation du numéro atomique moyen de la cible dans cette région.
\end{abstract}

\begin{abstract}
A scanning electron microscope supplied with a discharge system for the observation of insulating samples has permit to observe $\mathrm{LiF}$ crystals bombarded with $2 \mathrm{MeV}$ protons and $56 \mathrm{MeV}$ chlorine ions. The irradiated zone shows a clear contrast which allows a direct measure of the penetration depth of the incident ions. This contrast has been attributed to the $F$ and $F$-agglomerate centers which are also revealed by means of optical absorption measurements. The implanted ions can also be revealed in the stopping zone by means of the chemical contrast due to the variation of the mean atomic number of the target in this region.
\end{abstract}

1. Introduction. - Les cristaux d'halogénures alcalins bombardés avec des ions énergétiques se colorent. Les défauts créés qui sont responsables de cette coloration peuvent être classés en trois grandes catégories :

- les défauts anioniques du type $\mathrm{F}$ et du type $\mathrm{V}$ ainsi que leurs divers agglomérats ;

- les défauts cationiques du type centre I dans LiF [1];

- les centres colloïdaux intrinsèques qui résultent de l'agglomération des interstitiels anioniques ou cationiques et les centres colloïdaux extrinsèques qui peuvent provenir de la précipitation des ions implantés eux-mêmes [2].

Tous ces défauts peuvent être révélés facilement par des mesures d'absorption optique.

Nous savons que les défauts anioniques dans les halogénures alcalins peuvent être créés par des mécanismes d'excitation des ions halogènes [3]. Une étude précédente $[4,5]$ a montré que la création des centres du type $\mathrm{F}$ était bien reliée à la perte d'énergie par processus électronique des ions incidents. Concernant les défauts cationiques (centre I dans LiF), ils sont créés par choc direct entre l'ion incident et les ions alcalins de la cible.
Les pertes d'énergie par collisions élastiques étant prépondérantes pour des ions de basse énergie, les défauts cationiques seront localisés dans une zone très étroite située en fin de parcours des ions incidents. Cette zone de choc est très voisine de la zone d'implantation des ions incidents et on trouvera donc également dans cette région les défauts colloïdaux intrinsèques et extrinsèques. L'étude en absorption optique de cristaux bombardés avec des ions de basse énergie $(\sim 1 \mathrm{MeV})$ est rendue difficile par la complexité de cette zone et les grandes concentrations de défauts qui s'y trouvent. Dans ce cas, nous avons donc fait appel à une technique d'étude par microscopie électronique à balayage qui permet une observation directe de la zone de fin de parcours.

2. Technique d'irradiation et d'observation des cristaux. - Les cibles utilisées sont des lamelles monocristallines obtenues par clivage à partir de blocs provenant de Quartz et Silice. Elles ont été irradiées avec des protons de $2 \mathrm{MeV}$ provenant de l'accélérateur Van de Graaf de l'Institut de Physique nucléaire de Lyon et des ions $\mathrm{Cl}^{8+}$ de $56 \mathrm{MeV}$ provenant de l'accélérateur tandem du Centre d'Etudes Nucléaires de Saclay. 
Les doses d'ions incidents étaient de l'ordre de $5 \times 10^{16}$ ions par $\mathrm{cm}^{2}$. Après irradiation les échantillons sont clivés perpendiculairement à la face (100) bombardée et l'observation au microscope à balayage se fait suivant la direction de pénétration des ions incidents.

L'observation d'échantillons isolants en microscopie électronique à balayage est rendue difficile car la surface du cristal se charge sous l'impact du faisceau d'électrons.

En effet, le coefficient d'émission secondaire de la plupart des isolants est inférieur à l'unité lorsque l'énergie des électrons du faisceau d'observation est de l'ordre de 20 à $30 \mathrm{keV}$. La conductivité n'étant pas suffisante pour drainer les électrons supplémentaires vers le porteéchantillon, la résolution et la profondeur de champ diminuent rapidement au cours de l'observation, puis l'image devient plus profondément altérée par des surintensités locales instables.

En général, les isolants peuvent être observés :

- soit à basse énergie, mais dans ce cas la résolution est médiocre ;

- soit après avoir déposé sur la surface une mince couche métallique mais alors seul le contraste topographique est accessible.

Pour éviter ces différents inconvénients, nous avons réalisé un dispositif qui permet de décharger la surface en utilisant le faisceau d'électrons lents d'un canon auxiliaire [6, 7].

Pour de tels électrons de faible énergie $(\sim 1 \mathrm{keV})$ le coefficient d'émission secondaire est supérieur à 1 et dans ce cas, les charges apportées par le faisceau d'observation peuvent être compensées par un départ équivalent de charges sous l'impact du faisceau électronique auxiliaire.

Un tel dispositif a été monté sur un microscope à balayage Cambridge $M K 2$. Le positionnement du faisceau de décharge sur la zone à observer se fait à l'aide d'un microscope optique en utilisant la cathodoluminescence de l'échantillon. La détection électronique a été modifiée afin d'éliminer les perturbations de l'image dues au faisceau de décharge. Ainsi pour une observation visuelle, la détection est interrompue toutes les deux lignes et la décharge s'effectue pendant la deuxième ligne de balayage alors que pour un enregistrement photographique, chaque point de l'échantillon reçoit alternativement les deux faisceaux.

Cet appareil a été utilisé pour l'observation de nombreux isolants et les résultats obtenus ont confirmé son efficacité [8].

3. Résultats expérimentaux. - Les échantillons de LiF bombardés avec des protons de $2 \mathrm{MeV}$ et des ions chlore de $56 \mathrm{MeV}$ ont été observés immédiatement après irradiation et également après des traitements thermiques. Simultanément, l'évolution des défauts présents dans l'échantillon a été suivie par l'intermédiaire des spectres d'absorption optique.

\subsection{ECHANTILLONS BOMBARdÉS AVEC DES PROTONS} DE $2 \mathrm{MeV}$. - Les cristaux de LiF observés ont été fortement irradiés $\left(\sim 5 \times 10^{16}\right.$ protons $\left./ \mathrm{cm}^{2}\right)$. Le spectre d'absorption optique d'un tel cristal (Fig. 1a) indique que les concentrations de défauts sont très importantes $\left(\sim 10^{20}\right.$ défauts $\left./ \mathrm{cm}^{3}\right)$. L'observation correspondante au microscope à balayage montre un contraste clair dans toute la zone de pénétration des ions incidents (Fig. 1b). Ce contraste pourrait être

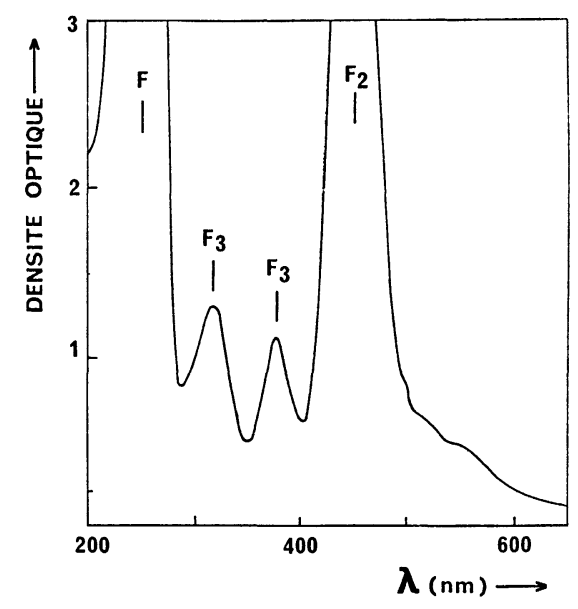

(a)

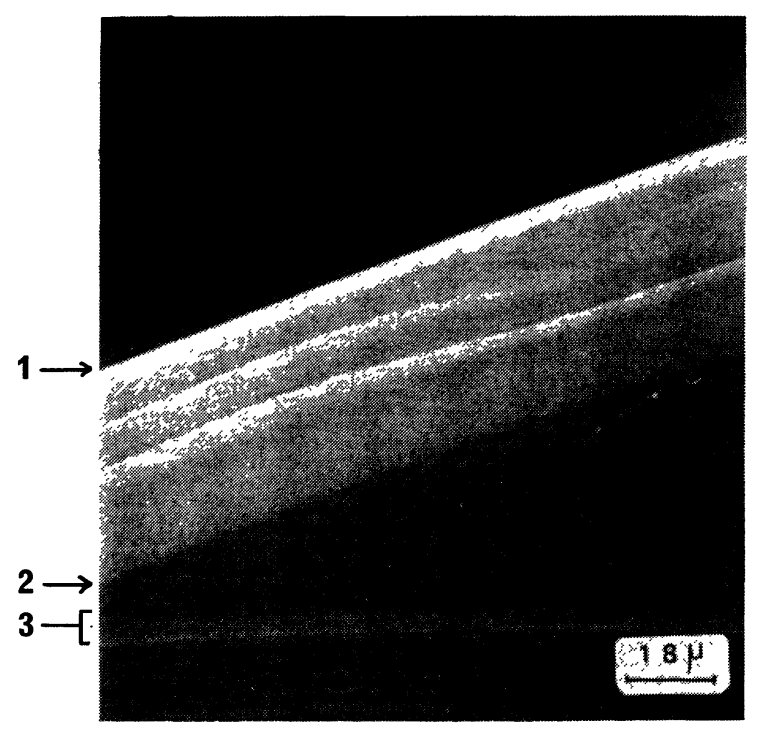

(b)

FIG. 1. - Echantillon de LiF bombardé avec $5 \times 10^{16}$ protons de $2 \mathrm{MeV}$ par $\mathrm{cm}^{2}: a$ ) Spectre d'absorption optique ; $b$ ) Photographie au microscope électronique à balayage : - Surface de l'échantillon [1]. - Fin de parcours des ions incidents [2]. - Perturbation due au réglage de la luminosité pour l'enregistrement photographique [3].

attribué à la présence des grandes concentrations de Centres $F$ et agglomérats de $F$ qui sont facilement ionisables et qui augmenteraient le nombre d'électrons secondaires émis. 
Après un traitement thermique à $300^{\circ} \mathrm{C}$ pendant 30 min., le contraste clair diminue et parallèlement le spectre d'absorption optique montre la décroissance des bandes d'absorption liées aux Centres F et agglomérats de $\mathrm{F}$.

Finalement, pour un traitement thermique à $350^{\circ} \mathrm{C}$, pendant $30 \mathrm{~min}$., les Centres du type $\mathrm{F}$ ont presque totalement disparu (Fig. $2 a$ ) et il subsiste vers $450 \mathrm{~nm}$ une bande d'absorption due aux colloïdes de Lithium [9, 10]. L'observation au microscope à balayage montre que le contraste clair a totalement disparu et vers la fin de parcours des ions incidents nous observons une zone de contraste sombre (Fig. $2 b$ ).

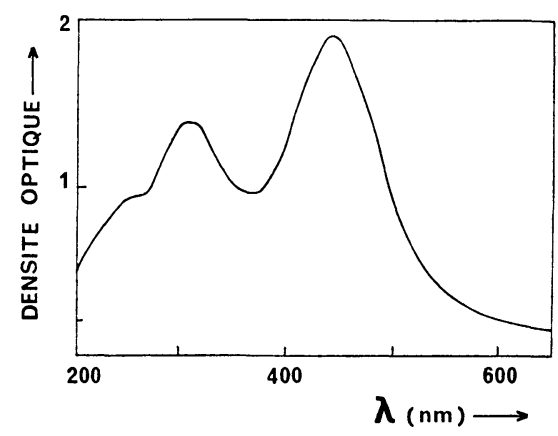

(a)

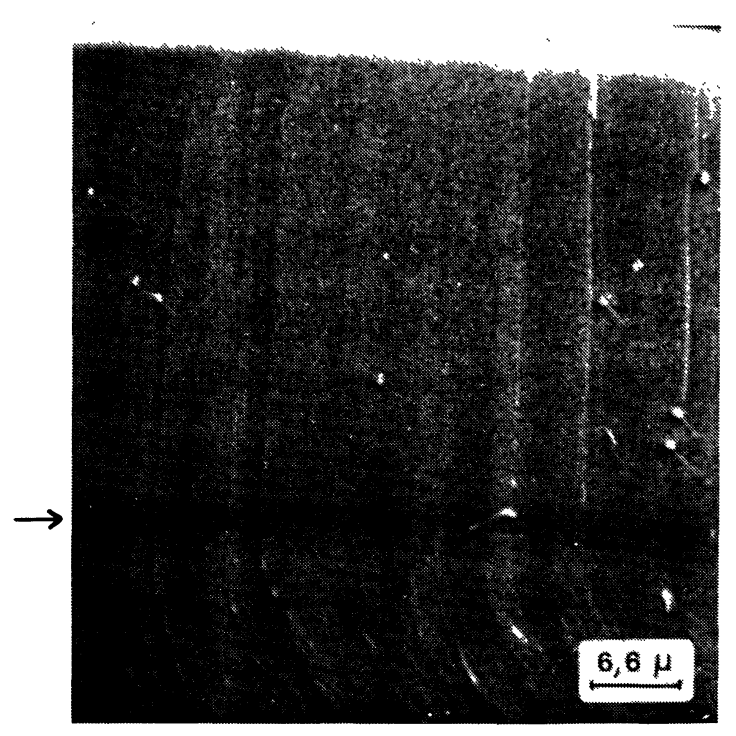

(b)

FIG. 2. - Echantillon de LiF bombardé avec $5 \times 10^{16}$ protons de $2 \mathrm{MeV}$ par $\mathrm{cm}^{2}$ et recuit à $300^{\circ} \mathrm{C}$ pendant $30 \mathrm{~min}$. puis $350^{\circ} \mathrm{C}$ pendant $30 \mathrm{mn}: a)$ Spectre d'absorption optique ; b) Photographie au microscope électronique à balayage.

La présence de cette zone sombre en fin de parcours semble indiquer une diminution du numéro atomique moyen de l'échantillon dans cette région. En fait la présence des protons implantés devrait donner une variation en sens inverse, toutefois, la variation du taux de rétrodiffusion due aux protons implantés est négligeable. Le contraste pourrait donc être attribué à un départ d'ions fluor dans cette zone de fin de parcours très perturbée. Nous savons qu'à la température ambiante, les interstitiels halogènes diffusent dans le cristal pour s'agglomérer [11]. Pendant les traitements thermiques, les atomes d'halogène pourraient atteindre la surface et ceci serait facilité par la grande perturbation du réseau qui règne en fin de parcours.

Signalons également que l'observation en microscopie à balayage permet une mesure directe de la profondeur de pénétration des ions incidents. Dans le cas des protons nous trouvons $36 \mu$, ce qui est en bon accord avec le parcours calculé à partir de la perte d'énergie électronique $(35 \mu)$.

3.2 ECHANTILLONS BOMBARDÉS AVEC DES IONS CHLORE DE $56 \mathrm{MeV}$. - La figure $3 a$ montre le spectre d'absorption optique d'un échantillon de LiF bombardé avec $5 \times 10^{16} \mathrm{Cl}^{8+} / \mathrm{cm}^{2}$. Les bandes d'absorption sont moins importantes que dans le cas précédent ce qui semble indiquer des concentrations de défauts plus faibles. En fait, la densité d'énergie dissipée par les

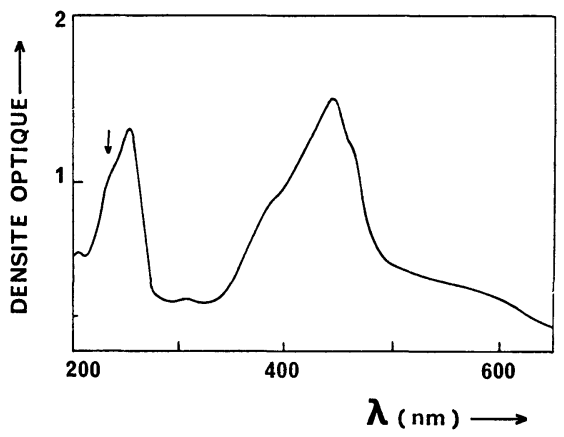

(a)

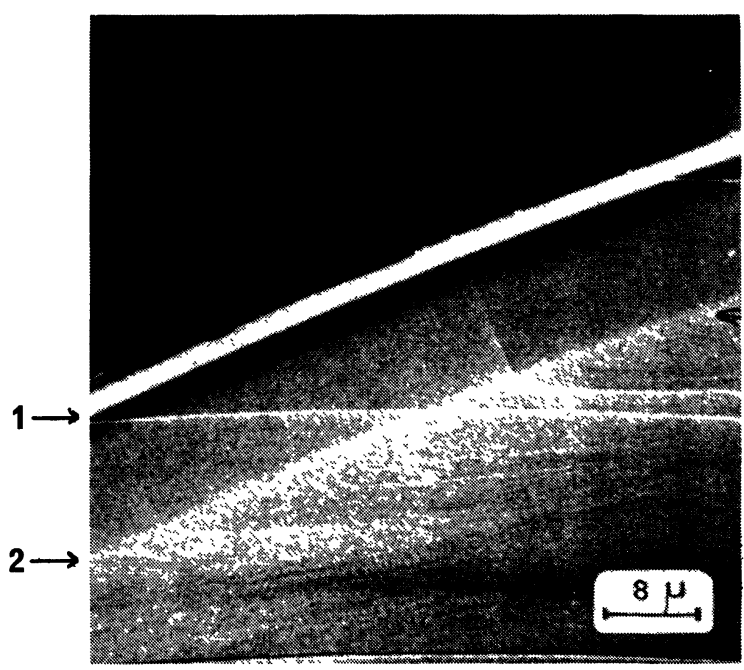

(b)

FIG. 3. - Echantillon de LiF bombardé avec $5 \times 10^{16}$ ions $\mathrm{Cl}^{8+}$ de $56 \mathrm{MeV}$ par $\left.\mathrm{cm}^{2}: a\right)$ Spectre d'absorption optique ; b) Photographie au microscope électronique à balayage : - Surface de l'échantillon [1]. - Zone d'implantation des ions chlore [2]. 
ions chlore est bien supérieure à celle des protons et un effet de saturation et de redestruction des défauts est probable. La bande d'absorption située vers $230 \mathrm{~nm}$ peut être attribuée au chlore implanté dans le réseau [5].

L'observation au microscope à balayage d'un tel échantillon (Fig. $3 b$ ) montre la présence d'une bande de contraste clair en fin de parcours des ions incidents. Ce contraste peut être attribué aux ions chlore implantés. D'après la variation du taux de rétrodiffusion, en fonction du numéro atomique donné par Weinryb et Philibert [12], nous constatons que le taux de rétrodiffusion du chlore $(Z=35)$ est 5 fois supérieur à celui de $\operatorname{LiF}(Z$ moyen $=6)$. Pour $5 \times 10^{16}$ ions chlore implantés sur une épaisseur de $1 \mu$, nous obtenons une concentration de $0,8 \%$, ce qui donnerait une variation du signal d'environ $4 \%$.

Cette valeur est en bon accord avec le contraste observé expérimentalement. La profondeur de pénétration des ions chlore mesurée sur la figure $3 b$ est d'environ $12 \mu$. La largeur de la zone d'implantation étant d'environ $1 \mu$, ceci donnerait un straggling $\Delta R / R$ de $8 \%$.
Le straggling calculé à partir de la théorie de Lindhard [13] est de $10 \%$.

4. Conclusion. - La microscopie électronique à balayage appliquée à des cristaux isolants tels que les halogénures alcalins peut permettre une étude de la création de défauts par bombardement ionique. Les ions implantés peuvent être révélés par l'intermédiaire du contraste chimique qui apparaît en fin de parcours. Cette technique sera donc très intéressante pour l'étude de défauts tels que les colloïdes extrinsèques qui proviennent de l'agglomération des ions implantés. Signalons également que le dispositif permet l'observation des pseudo-lignes de Kikuchi ce qui permettra l'étude de la déformation du réseau cristallin dans la zone irradiée.

Remerciements. - Nous remercions Monsieur le Professeur A. Sarazin et Monsieur B. Delaunay pour leur assistance avec les accélérateurs Van de Graaff de l'I. P. N. de Lyon et tandem du C. E. N. de Saclay.

\section{Bibliographie}

[1] Farge, Y., Lambert, M. et Guinier, A., J. Phys. Chem. Solids 27 (1966) 499.

[2] Davenas, J., Perez, A., Thevenard, P. et Dupuy, C. H. S. Phys. State Sol. (a) 19 (1973) 679.

[3] Pooley, D., Proc. Phys. Soc.; 87 (1966) 245.

[4] Perez, A., Thèse Lyon, 1974.

[5] Thevenard, P., Thèse Lyon, 1974.

[6] Morin, P., Pitaval, M. et Vicario, E., J. Phys. E. (Scientific Inst.) to be published (1975).

[7] Vicario, E. et Morin, P., Brevet Anvar nº 7432368, 16 septembre 1974.
[8] Morin, P., Thèse de spécialité, Lyon, 1974.

[9] Farge, Y., Thèse Paris, 1967.

[10] Radchenko, I. S., Sov. Phys. Solid State 11 (1970) 1476.

[11] Durand, P., Farge, Y. et Lambert, M., J. Phys. Chem. Solids 30 (1969) 1953.

[12] Weinryb, E. et Philibert, J., C. R. Hebd. Séan. Acad. Sci. 258 (1964) 4535.

[13] Nelson, R. S., The observation of atomic collisions in crystalline solids (North-Holland Publ. Co.) 1968, p. 42. 